\title{
Perforated Pantograph Horn Aeolian Tone Suppression Mechanism
}

\author{
Mitsuru IKEDA \\ Senior Researcher, Current Collection Lab., \\ Railway Dynamics Div.
}

\author{
Takehisa TAKAISHI \\ Assistant Senior Researcher, Aerodynamics \& Acoustics Lab., \\ Environmental Engineering Div.
}

An Aeolian tone suppression mechanism of a perforated pantograph horn has been experimentally studied with an anechoic wind tunnel. Three types of horn models - a simple cylinder, a cylinder perforated with periodic holes and one with a continuous slit - were used to compare aerodynamic noise characteristics and the flow field around them. Experimental results showed that the wake shear layers of the perforated cylinder become stable due to the jets emitted from the holes at regular intervals, thus providing the optimal way of suppressing the Aeolian tone. In the case of the cylinder with a continuous slit, the Aeolian tone was also suppressed, but the interaction between the continuous jet and the shear layer caused another narrow-band noise.

Keywords: Aerodynamic noise, Aeolian tone, Pantograph, Horn, Wayside noise

\section{Introduction}

The trackside noise emitted by high-speed trains is caused mainly by aerodynamic effects. The noise generated by a pantograph, which is one of the main noise sources on a train set, is also dominated by aerodynamic noise in the high-speed range. Hence various measures have been developed to reduce pantograph aerodynamic noise.

A low-noise horn is one of such measures. The pantograph horn, which is installed on both side-ends of the panhead to ensure movement onto the contact strip of out-of-running wire, is formed by a bow-shaped cylinder. Therefore, the horn tends to generate a conspicuous narrow-band noise, known as the Aeolian tone. Caused by the Karman vortex street shed from the body, the Aeolian tone is one of the most frequently encountered aerodynamic noises. The low-noise horn, perforated with periodic holes along its axis as shown in Fig. 1, is remarkably successful in suppressing this phenomenon. These periodic holes are parallel in the direction of travel.

Figure 2 shows the aerodynamic noise source distribution on a low-noise pantograph measured with an Xarray microphone. This pantograph has a low-noise horn mounted on one side of the panhead, and a conventional horn without holes on the other. It can be seen that less aerodynamic noise was generated by the low-noise horn, which was thus able to make a discernible contribution to trackside noise reduction.

As results of noise reduction efforts, it has been discovered that the dominant aerodynamic noise source of a low-noise type pantograph is the broadband noise generated by the panhead itself; the conspicuous Aeolian tone being no longer generated in the case of the low-noise type pantograph. Therefore, reduction of the broadband noise has been strongly needed in order to reduce trackside noise even further. Of course, we have a classic method of reducing broadband aerodynamic noise, which is to make the panhead shape smoother aerodynamically, but we have not yet obtained a quantitative principle to reduce the broadband noise. We believe that it is very

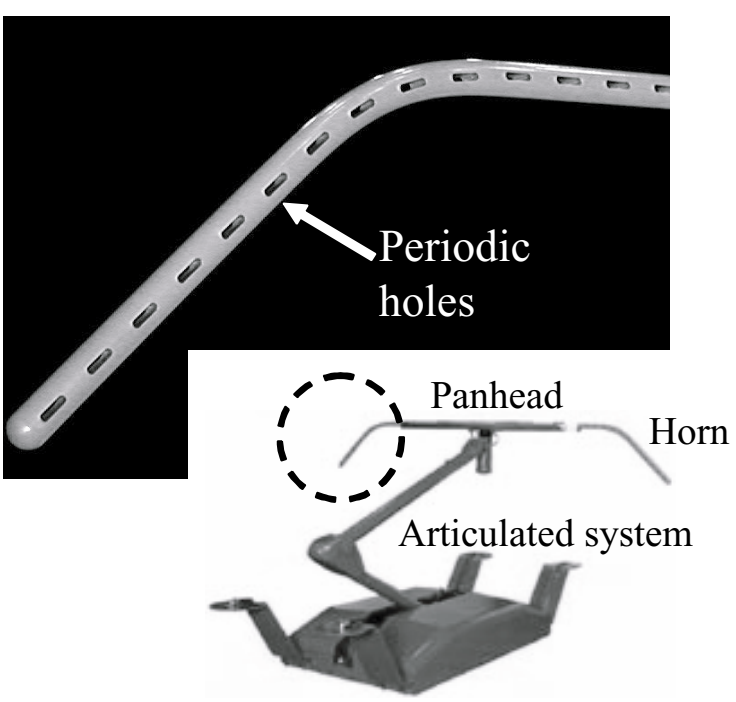

Fig. 1 Low-noise pantograph horn

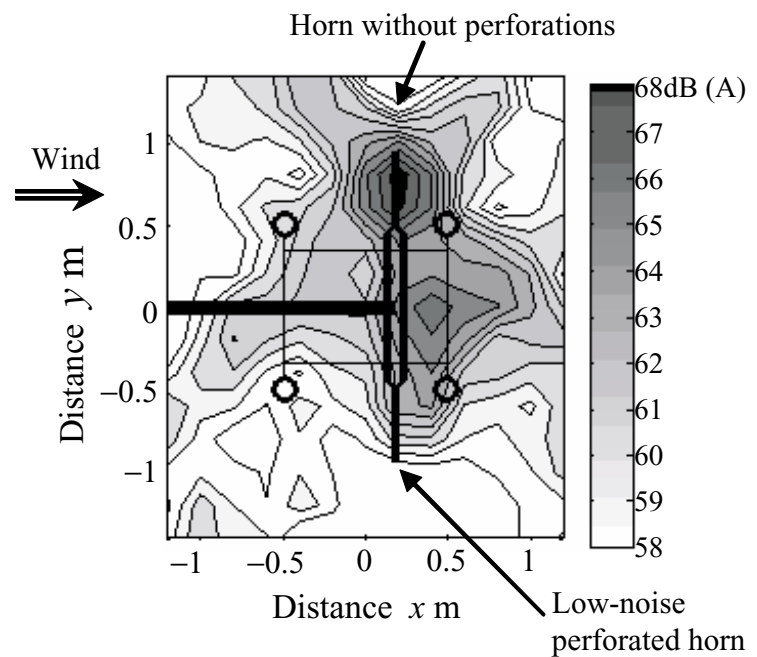

Fig. 2 Noise source distribution on a low-noise pantograph measured with an X-array microphone

(PEGASUS: $200 \mathrm{~km} / \mathrm{h} 1250 \mathrm{~Hz}$ ) 
important to have a farther understanding of the mechanism that generates the aerodynamic noise, in order to achieve drastic noise reduction.

In this study, therefore, we investigate an Aeolian tone suppression mechanism using the low-noise horn. We performed wind tunnel tests by using a two-dimensional model representing the perforated low-noise horn. In this paper, we report on the experimental results of the wind tunnel tests and propose a mechanism to suppress the Aeolian tone of a perforated pantograph horn.

\section{Experimental description}

\subsection{Test models}

In order to compare their aeroacoustic and aerodynamic properties, three types of horn model - a simple cylinder, a cylinder perforated with periodic holes, and a cylinder with a continuous slit - were investigated by means of wind tunnel tests. The cylinder with a continuous slit was tested to clarify the difference between the effect of the perforations and that of a continuous slit. These models have a two-dimensional configuration to simplify the aeroacoustic phenomenon, whereas a real pantograph horn has a bow-shaped configuration. Therefore, it was confirmed beforehand that there was no essential difference between the aeroacoustic characteristics of the cylinder and those of a bow-shaped cylinder either with or without perforations.

Figure 3 shows the model designs. The diameter $(D)$ of these models is $50 \mathrm{~mm}$, and the span length $(L)$ is $600 \mathrm{~mm}$. The real horn, which is used at speeds up to $300 \mathrm{~km} / \mathrm{h}$, has a diameter of $25 \mathrm{~mm}$. In the present experiment, the flow velocity $\left(U_{\infty}\right)$ was basically set at $41.7 \mathrm{~m} / \mathrm{s}(150 \mathrm{~km} / \mathrm{h})$. Therefore, the Reynolds number in this experiment matched that of an operational horn traveling at $300 \mathrm{~km} / \mathrm{h}$. When the diameter of the model is taken as the scaling length, the Reynolds number is 1.1 $\times 10^{5}$. Each perforation is rectangular, $40 \mathrm{~mm} \times 10 \mathrm{~mm}$ in size, and arranged at $80 \mathrm{~mm}$ intervals. The dimensions of the continuous slit are $600 \mathrm{~mm} \times 10 \mathrm{~mm}$.
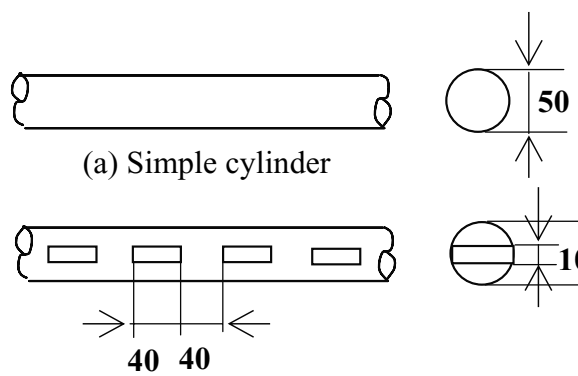

(b) Perforated cylinder

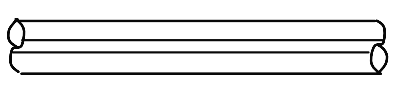

(c) Cylinder with continuous slit
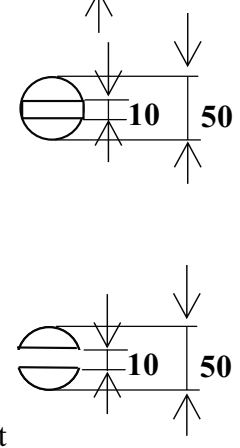

Fig. 3 Horn models designs

( Span length: 600mm )

\subsection{Experimental setup}

In this study, we performed wind tunnel tests by using RTRI's small-scale anechoic wind tunnel, which is of the single return type with an open test section. The test section, the cross section of which measures $720 \mathrm{~mm}$ wide $\times 600 \mathrm{~mm}$ high, is located inside the anechoic room and has a maximum flow velocity of $42 \mathrm{~m} / \mathrm{s}(150 \mathrm{~km} / \mathrm{h})$.

Figure 4 shows the experimental apparatus in the wind tunnel. The model was mounted vertically $500 \mathrm{~mm}$ downstream of the nozzle tip with its center set as the origin of the coordinates, the main flow direction as the $x$-axis, side direction as the $y$-axis, and spanwise direction as the $z$-axis. In the case of the perforated cylinder model, the origin of the $\mathrm{z}$-axis corresponded to the center of a hole.

In order to maintain a two-dimensional flow in the test section, end-walls were installed on both sides of the test section, as shown in Fig. 4. These end-walls were $1,200 \mathrm{~mm}$ wide and $1,500 \mathrm{~mm}$ long. The lower wall was made of a $10 \mathrm{~mm}$-thick acrylic plate in order to use a Particle Image Velocimetry (PIV) system. On the other hand, the upper wall was made of a $7 \mathrm{~mm}$-thick acoustically transparent plate (Polyvinyl Alcohol sponge) to prevent sound reflection on it. By using these types of wall, we were able to measure the aerodynamic noise emitted from the model accurately with no interference from sound reflection by using a microphone set at the level of the lower wall.

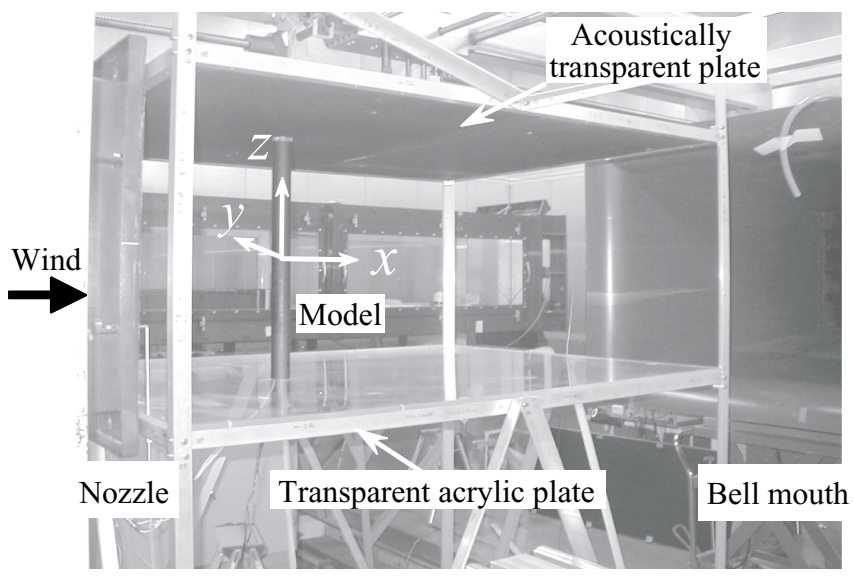

Fig. 4 Horn model in the wind tunnel

\subsection{Measurement devices}

Aerodynamic noise, static surface pressure and flow field were measured in these tests. The aerodynamic noise of the model was measured at $(x, y, z)=(0,-2000 \mathrm{~mm}$, $-300 \mathrm{~mm}$ ) with a $1 / 2$-inch RION UC-31 condenser microphone. The static surface pressures at 24 pressure taps on the model were also measured. The reference pressure $p_{\infty}$ was measured at $(x, y, z)=(-450 \mathrm{~mm}, 0 \mathrm{~mm}$, $-300 \mathrm{~mm}$ ) on the lower wall. Moreover, in order to measure the fluctuating pressures on the model surface, Kulite XT-190 and LQ-125 pressure transducers were installed.

The velocity field around the model was measured by using the 2D-Type PowerView particle image velocimetry 
(PIV) system manufactured by TSI. The resolution of this system is $2,048 \times 2,048$ pixels, and its dynamic range is 12bit. The maximum energy of $\mathrm{Nd}$ :YAG laser is $200 \mathrm{~mJ}$ per pulse and its repetition rate was set at $4 \mathrm{~Hz}$. The flow field measurement was repeated 250 times, and the average of these results calculated as an ensemble average.

\section{Experimental results}

\subsection{Visualization of the flow field in the wake}

With usage of the smoke method, we carried out flow visualization tests by using exclusive models, which were the same scale of the real pantograph horn. The velocity of the free stream was set at $4.2 \mathrm{~m} / \mathrm{s}$.

Figure 5 shows the visualization test results. The distinct Karman vortexes being shed from the simple cylinder are clearly visible. In the case of the perforated cylinder, only weak vortexes can be observed in the wake. However, we can observe a conspicuous arrow-like jet being ejected from a hole into the wake.

Similarly to the perforated cylinder, no distinct largescale vortexes can be observed in the wake of the cylinder with a continuous slit, and a jet is also being ejected from it. However, the jet does not blow out like an arrow but leans toward either of the separated shear layers, indicating that it is unstable.

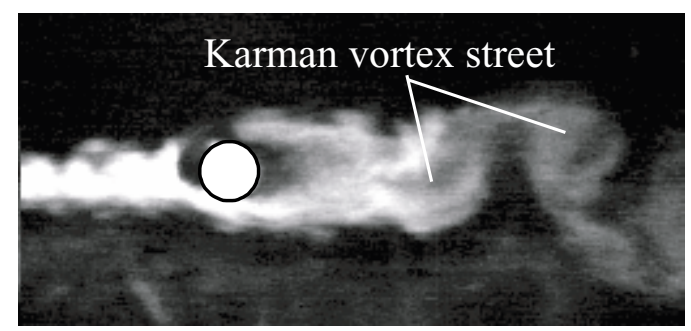

(a) Simple cylinder

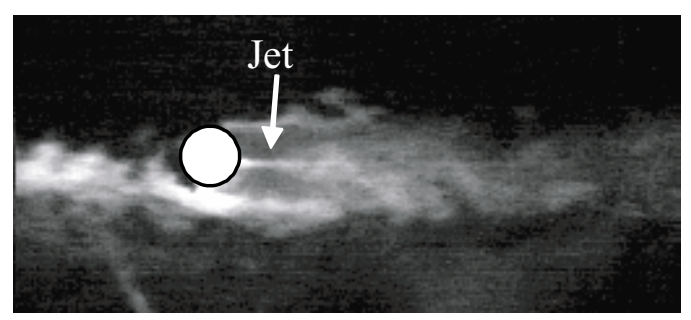

(b) Perforated cylinder

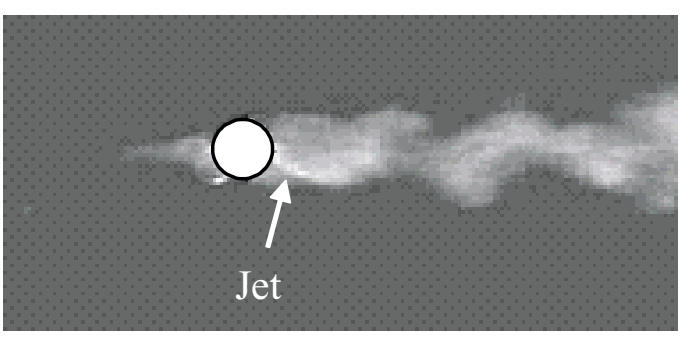

(c) Cylinder with continuous slit

Fig. 5 Visualized model wake flow patterns

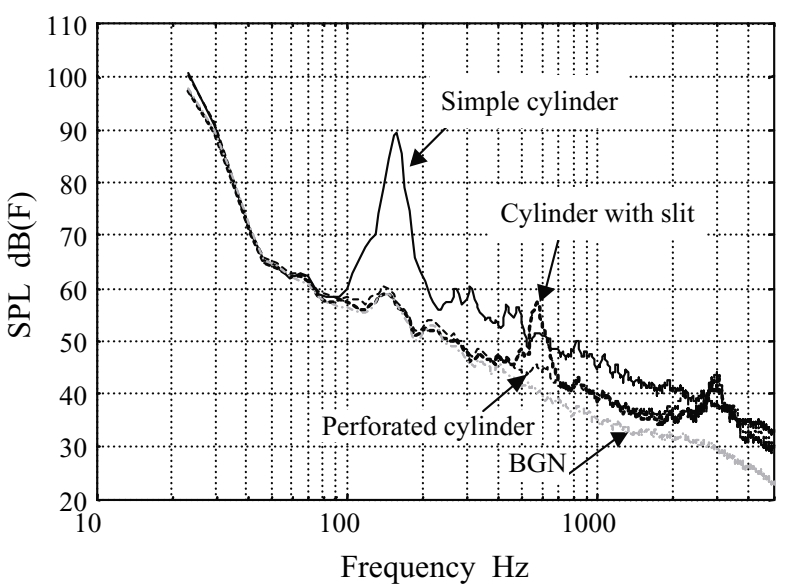

Fig. 6 Sound pressure level comparison at

$(x, y, z)=(0 \mathrm{~mm},-2000 \mathrm{~mm},-300 \mathrm{~mm})(150 \mathrm{~km} / \mathrm{h})$

\subsection{Aerodynamic noise}

Figure 6 compares sound pressure levels among the three models measured at $150 \mathrm{~km} / \mathrm{h}$. A conspicuous narrow-band noise is generated at $160 \mathrm{~Hz}$ in the case of the simple cylinder. The Strouhal number of this noise is about 0.19 , which corresponds to that of the Aeolian tone caused by a cylinder. In the cases of both the perforated cylinder and the cylinder with a continuous slit, however, a moderate peak arises at around $3 \mathrm{kHz}$ instead of the Aeolian tone. The frequency of this noise does not vary even if the flow velocity changes. Therefore, this noise is produced by acoustic resonance at the holes or the slit. In fact, the wavelength of this narrow-band noise is $110 \mathrm{~mm}$, which is almost twice the length of the holes or the slit in the $x$-axis. Furthermore, we also observe another narrow-band noise around $600 \mathrm{~Hz}$ in the case of the cylinder with a slit, the frequency of which changes in proportion to the flow velocity. Therefore, this narrowband noise having a peak around $600 \mathrm{~Hz}$ depends on the aerodynamic characteristics of the wake. Except for this narrow-band noise, the sound pressure level of the perforated cylinder and that of the cylinder with a slit are lower than that emitted from the simple cylinder.

\subsection{Model static surface pressure distribution}

Figure 7 shows the measurement results from timeaveraged static surface pressure distribution tests carried out on the models. The free stream velocity was set at $150 \mathrm{~km} / \mathrm{h}$. The transverse axis of this figure indicates $\theta$, or the counterclockwise angle from the front stagnation point.

The pressure profile of the simple cylinder shown in Fig. 7 (a) indicates that flow separation occurs near the location where the angle $\theta$ is 80 degrees, and the base pressure coefficient is about -1.2 . These properties involve features of laminar separation. In fact, the Reynolds number of this flow is $1.1 \times 10^{5}$, at which laminar separation normally occurs around a cylinder.

Figure 7 (b) shows the pressure profile of the perforated cylinder. The pressure at the cross section containing a hole $(z=-80 \mathrm{~mm})$ is almost the same as that at the 


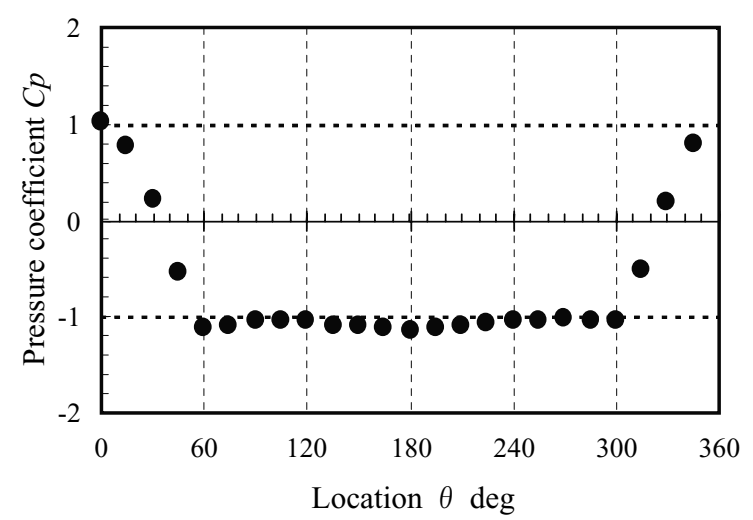

(a) Simple cylinder

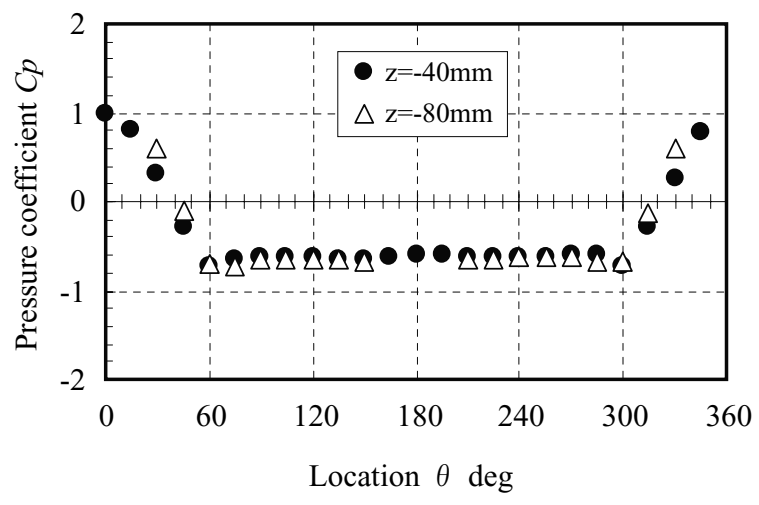

(b) Perforated cylinder

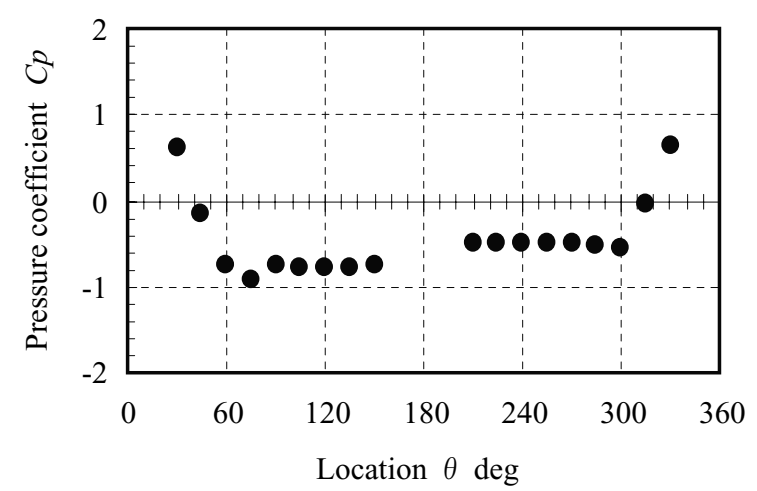

(c) Cylinder with continuous slit

Fig. 7 Static surface pressure coefficients of models $(150 \mathrm{~km} / \mathrm{h})$

cross section without a hole $(z=-40 \mathrm{~mm})$. Therefore, the holes have an effect not only on the flow nearby but also on the wake as a whole. The separation point on the perforated cylinder is close to that on the simple cylinder. However, the base pressure of the perforated cylinder has a higher value.

Figure 7 (c) shows the pressure profile of the cylinder with a continuous slit. As mentioned in section 3.1, the jet ejected from the slit is unstable and leans toward the separated shear layer. For this reason, the pressure profile around the cylinder with a continuous slit is asymmetric no matter how strictly the angle of attack of the cylinder is set at zero.

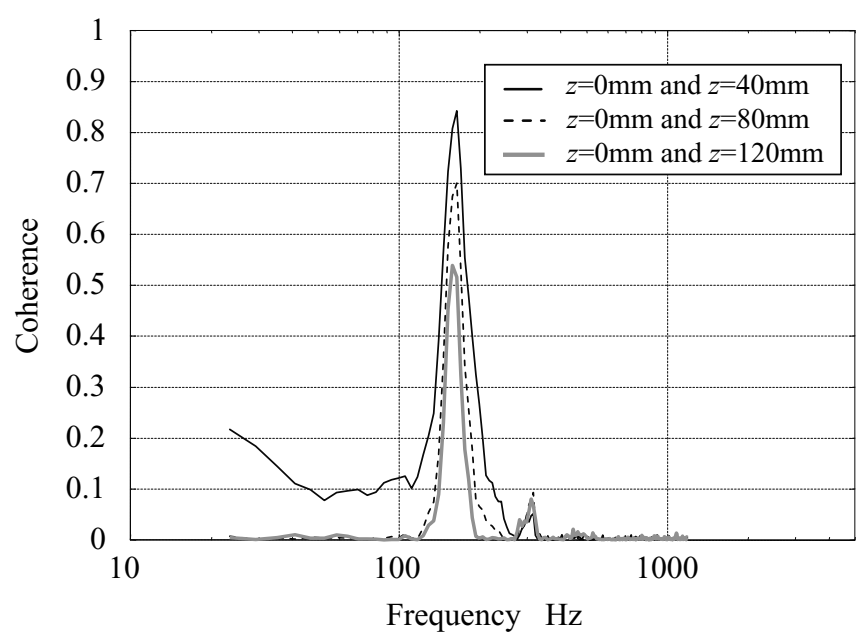

Fig. 8 Spanwise pressure coherence of simple cylinder $(150 \mathrm{~km} / \mathrm{h}: \theta=80 \mathrm{deg}$.)

\subsection{Spanwise coherence of fluctuating surface pressure}

By using pressure transducers located at $\theta=80 \mathrm{deg}$ on the model, the coherence among fluctuating pressures at $z=0 \mathrm{~mm}, z=40 \mathrm{~mm}, z=80 \mathrm{~mm}$, and $z=120 \mathrm{~mm}$ were calculated as shown in Fig. 8. This figure indicates that the spanwise coherence takes a high value at a frequency of $160 \mathrm{~Hz}$ and at its harmonic in the case of the simple cylinder; this frequency corresponding to the shedding frequency of the Karman vortexes. Therefore, the Karman vortex shed from the simple cylinder has a large two-dimensional structure in the spanwise direction. On the other hand, the values of spanwise coherence are not so large in the spanwise direction in the cases of the perforated cylinder or that with a continuous slit. This means that these cylinders do not have a large-scale flow structure in their wakes.

\subsection{Flow velocity distribution in the wake}

The flow velocity distribution in the wake of the models was measured in detail by using a PIV system in order to examine the flow structure. The laser emission interval of the PIV system was set at $15 \mu \mathrm{s}$. Figures 9 to 11 show the ensemble averaged flow field in the wake of the models at $150 \mathrm{~km} / \mathrm{h}$ : Fig. 9 shows the vorticity distribution on the $x y$-axis; Fig. 10 the distribution of the turbulence level in the direction of the $x$-axis; and Fig. 11 the distribution of the turbulence level in the direction of the $y$-axis, respectively. In these diagrams, each arrow indicates the velocity vector at each point.

In the case of the simple cylinder, we can observe that the turbulence level in the direction of the $y$-axis takes the highest value in the vicinity of $x=2 D$ to $2.5 D$, which is about $60 \%$. In this region, the shear layers separated from each side of the cylinder roll up alternatively. In the downstream side of this region, the mean flow velocity quickly recovers.

As for the perforated cylinder, the jet ejected from a hole reaches the point of $x=2 D$, and rapidly diffuses in the vicinity of $x=2.5 D$. The flow velocity in the wake increases gradually along the $x$-axis toward the downstream 


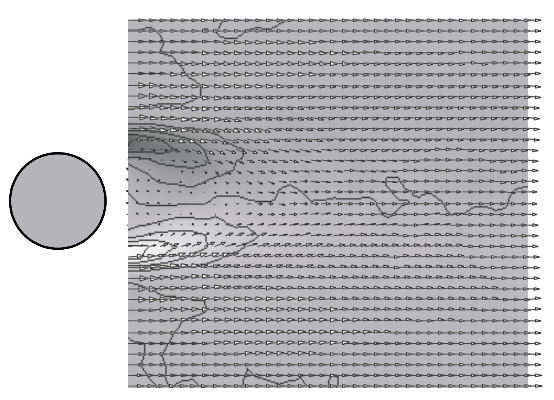

(a) Simple cylinder

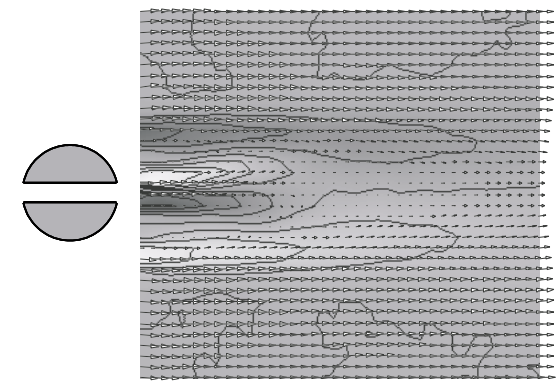

(b) Perforated cylinder $(z=0 \mathrm{~mm})$

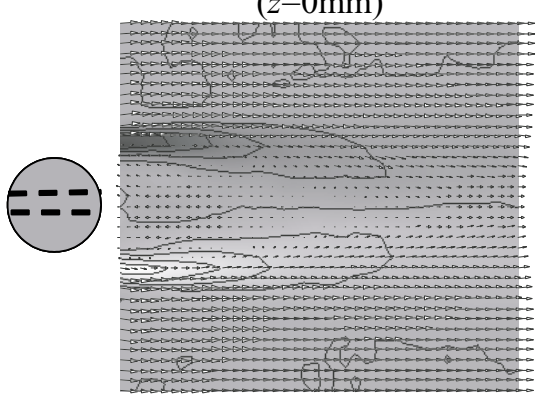

(c) Perforated cylinder $(z=-40 \mathrm{~mm})$

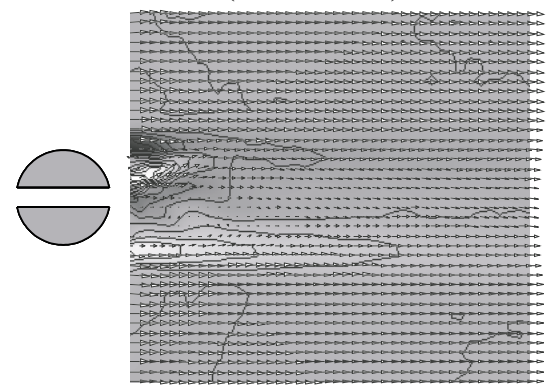

(d) Cylinder with slit

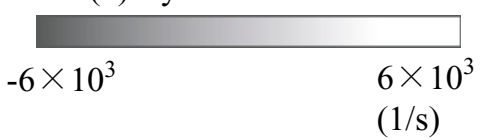

Fig. 9 Mean vorticity field $(150 \mathrm{~km} / \mathrm{h}$ xy-axis $)$

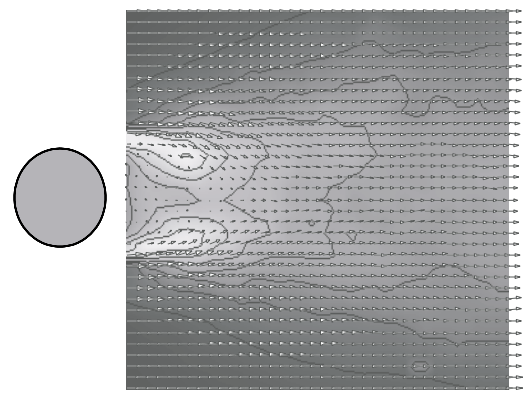

(a) Simple cylinder

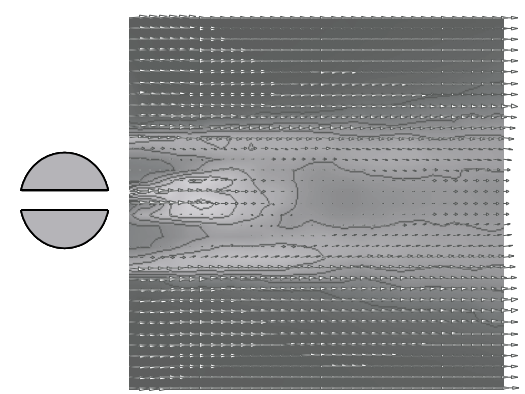

(b) Perforated cylinder $(z=0 \mathrm{~mm})$

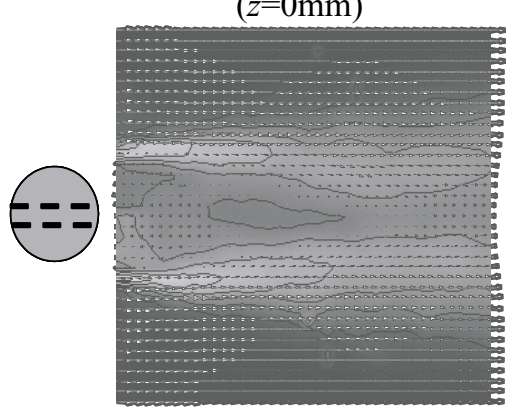

(c) Perforated cylinder $(z=-40 \mathrm{~mm})$

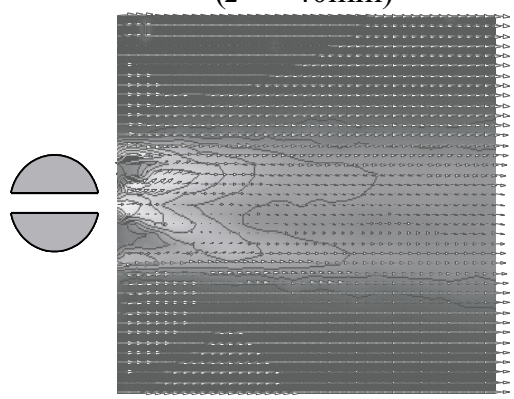

(d) Cylinder with slit

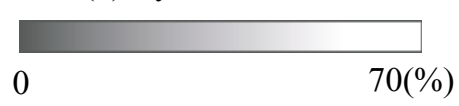

Fig. 10 Turbulence level in the $x$ direction $(150 \mathrm{~km} / \mathrm{h} x y$-axis $)$

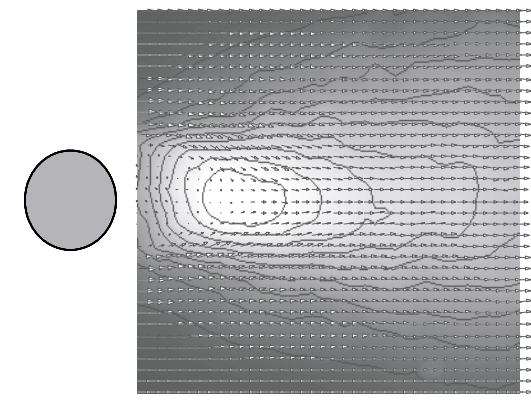

(a) Simple cylinder

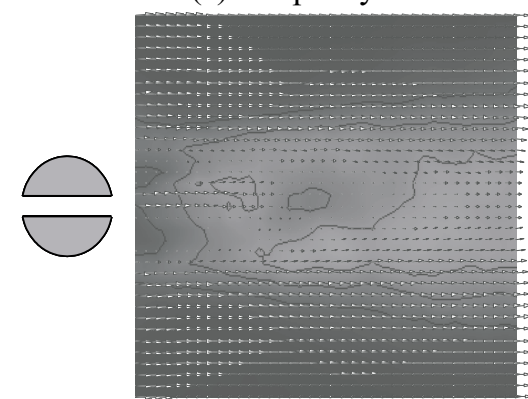

(b) Perforated cylinder $(z=0 \mathrm{~mm})$

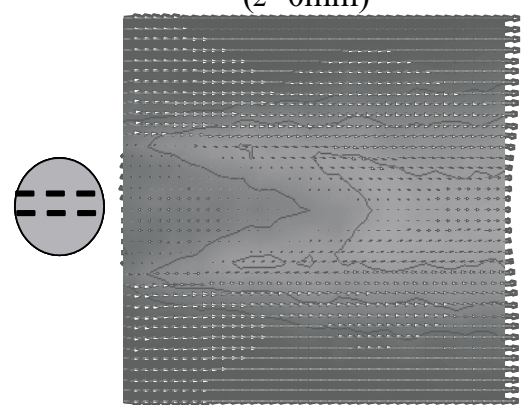

(c) Perforated cylinder $(z=-40 \mathrm{~mm})$

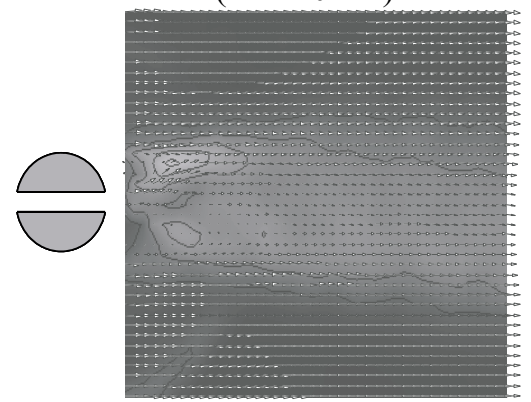

(d) Cylinder with slit

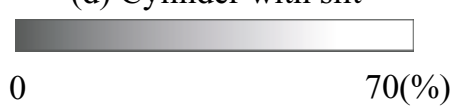

Fig. 11 Turbulence Level in the $y$ direction $(150 \mathrm{~km} / \mathrm{h} x y$-axis) side, and the width of the wake is almost constant until $x=6 D$. The turbulence level in the $y$-axis is quite low in the wake being compared with the case of the simple cylinder. Therefore, we can see that the separated shear layers are very stable in the case of the perforated cylinder. It is noticeable that the flow pattern of the perforated cylinder with holes at the cross section $(z=0 \mathrm{~mm})$ is almost similar to that at the cross section that contains no hole $(z=-40 \mathrm{~m})$ except for the existence of the ejected jet. This means that the ejected jet strongly diffuses in the spanwise direction.

The flow pattern of the cylinder with a continuous slit can be characterized by the ejected jet, which leans toward either of separated shear layers, and also by the existence of high turbulent region near the outlet of the slit where the $x$-directional turbulence level reaches high 
levels. Furthermore, the mean flow velocity in the wake recovers more rapidly than that of the perforated cylinder. These facts indicate that the jet ejected from the slit is unstable when compared with the jet ejected from a perforated hole. That is probably due to the two-dimensional configuration of a jet flow ejected from a two-dimensional slit.

\section{Discussion}

The measured results indicate that the Karman vortex pairs, which are shed from the simple cylinder into the wake and roll up each other, make the flow fluctuate strongly to generate a region where turbulence level in the $y$-direction reaches high values. A large fluctuating force acts on the cylinder along the $y$-axis due to frequent vortex shedding to cause the Aeolian tone. In contrast, the shear layers separated from the perforated cylinder are stable due to the jets, thereby no distinct roll up of the vortex layers is formed. Therefore, the Aeolian tone can barely be discerned in the case of the perforated cylinder.

The effect of providing the cylinder with perforations can be explained by Fig. 12. The large momentum injected into the wake by the jets ejected from the perforations restrains the separated shear layers and prevents them from interacting with each other. Therefore, the shear layers are stable and do not roll up each other. In addition, the jets are ejected at regular intervals and have a stable, three-dimensional structure.

Similarly, the shear layers separated from the cylinder with a continuous slit are stable due to the injection momentum of the jet. Therefore, the Aeolian tone can barely be detected. However, the two-dimensional jet ejected from the continuous slit is unstable, this providing a conspicuous narrow-band noise of $600 \mathrm{~Hz}$.

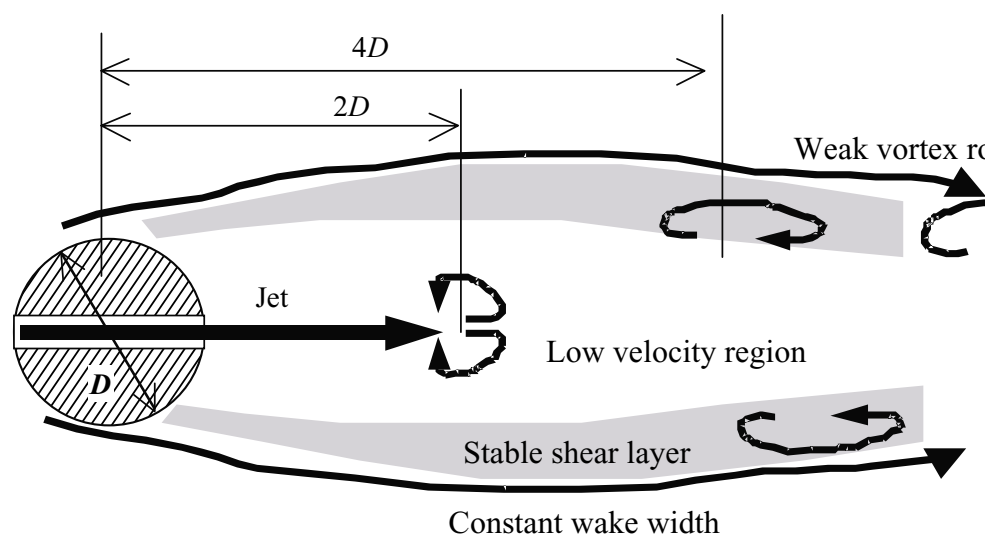

Fig. 12 Schematic perforated cylinder wake flow pattern
Hence, the momentum injected into the wake and the stabilization of the jets are essential factors in suppressing the Aeolian tone of the perforated pantograph horn. It appears that collapsing the spanwise flow structure by jet ejection has only a small effect on Aeolian tone suppression.

The perforations generate their own resonant noise, the frequency of which depends on their dimensions. When the contribution of the resonant noise cannot be ignored, the shape of the holes should be optimized to avoid strong acoustic resonance.

\section{Conclusion}

An Aeolian tone suppression mechanism of a perforated pantograph horn has been studied experimentally. The results of wind tunnel tests show that the momentum injected into the wake by the jets ejected from the holes and the stabilization of the ejected jet due to its three-dimensional structure stabilize the separated shear layers and prevent Aeolian tone generation. In the case of the cylinder with a continuous slit, the Aeolian tone is also suppressed, but a distinct narrow-band noise is caused by the interaction between the continuous jet and the shear layer.

\section{Acknowledgments}

We would like to extend our thanks to Drs. H. Fujita (Nihon Univ.), C. Kato (Tokyo Univ), and A. Iida (Kogakuin Univ.) for their invaluable contributions to this study.

\section{References}

1) Ikeda, M., "Study of the aerodynamic noise characteristics of bluff bodies as a pantograph member," Proceedings of Forum Acusticum Sevilla 2002, on CD-ROM, 2002.

2) Takaishi, T. et al: "Effect of periodic holes on the suppression of aeroacoustic noise from a pantograph horn," Proceedings of FEDSM '03, 4th ASME_JSME Joint Fluid Engineering Conference, 2003.

3) Fujita, H. et al: "The Aeolian tone characteristics of a circular cylinder in high Reynolds number flow," AIAA-99-1894, 1999. 